\title{
Existence and multiplicity of periodic solutions for some second-order Hamiltonian systems
}

\author{
Hongmin Suo ${ }^{1 *}$, Lan $\mathrm{Di}^{2^{*}}$, Yucheng $\mathrm{An}^{1,3}$ and Changmu Chu ${ }^{1}$
}

Dedicated to Professor SS Chang on his 80th birthday

${ }^{\text {*Correspondence: }}$
gzmysxx88@sina.com;
dilan126@163.com
'School of Sciences, Guizhou Minzu
University, Guiyang, 550025, China
${ }^{2}$ School of Digital Media, Jiangnan
University, Wuxi, Jiangsu 214122,
China
Full list of author information is
available at the end of the article

available at the end of the article

\begin{abstract}
The purpose of this paper is to study the existence and multiplicity of periodic solutions for the following non-autonomous second-order Hamiltonian systems: $\ddot{u}(t)=\nabla F(t, u(t))$ a.e. $t \in[0, T], u(0)-u(T)=\dot{u}(0)-\dot{u}(T)=0$, where $T>0$. Some new existence and multiplicity theorems are obtained by using the least action principle, and the minimax method in critical point theory, which unify and generalize some of the recent corresponding results in the literature.
\end{abstract}

Keywords: second-order systems; periodic solution; Sobolev's inequality; Wirtinger's inequality

\section{Introduction and main results}

Consider the second-order Hamiltonian systems

$$
\left\{\begin{array}{l}
\ddot{u}(t)=\nabla F(t, u(t)) \quad \text { a.e. } t \in[0, T], \\
u(0)-u(T)=\dot{u}(0)-\dot{u}(T)=0,
\end{array}\right.
$$

where $T>0$ and $F:[0, T] \times R^{N} \rightarrow R$ satisfies the following assumption:

(A) $F(t, x)$ is measurable in $t$ for every $x \in R^{N}$ and continuously differentiable in $x$ for a.e. $t \in[0, T]$, and there exist $a \in C\left(R^{+}, R^{+}\right), b \in L^{1}\left(0, T ; R^{+}\right)$such that

$$
|F(t, x)| \leq a(|x|) b(t), \quad|\nabla F(t, x)| \leq a(|x|) b(t)
$$

for all $x \in R^{N}$ and a.e. $t \in[0, T]$.

As is well known, a Hamiltonian system is a system of differential equations which can model the motion of a mechanical system. An important and interesting question is under what conditions the Hamiltonian system can support periodic solutions. During the past few years, under assumption (A) and some other suitable conditions, such as the coercivity condition, the convexity conditions, the sublinear nonlinearity conditions, the subquadratic potential conditions, the superquadratic potential conditions, the periodicity conditions, and even the type potential condition, and so on, the existence and multiplicity of periodic solutions are obtained for problem (1) in [1-20]. Inspired and motivated by

\section{Springer}

@2014 Suo et al.; licensee Springer. This is an Open Access article distributed under the terms of the Creative Commons Attribution License (http://creativecommons.org/licenses/by/2.0), which permits unrestricted use, distribution, and reproduction in any medium, provided the original work is properly cited. 
the results due to Wang and Zhang [1], Aizmahin and An [2], Ye and Tang [5], and Ma and Tang [9], we obtain some new existence theorems for problem (1), which generalize some results mentioned above.

The following main results are obtained by using the least action principle and by the minimax methods.

Theorem 1.1 Suppose that $F(t, s)=G(x)+H(t, x)$ satisfies assumption (A) and the following conditions:

(F1) there exist constants $C \geq 0, C^{*}>0$, and a positive function $q \in C\left(R^{+}, R^{+}\right)$with the properties:

(q1) $q(s) \leq q(t)+C, \forall s, t \in R^{+}$and $s \leq t$,

(q2) $q(s+t) \leq C^{*}(q(s)+q(t)), \forall s, t \in R^{+}$,

(q3) $t q(t)-2 Q(t) \rightarrow-\infty$ as $t \rightarrow+\infty$,

(q4) $Q(t) / t^{2} \rightarrow 0$ as $t \rightarrow+\infty$,

where $Q(t)=\int_{0}^{t} q(s) d s$. Moreover, there exist $f \in L^{1}\left(0, T ; R^{+}\right)$and $g \in L^{1}\left(0, T ; R^{+}\right)$such that

$$
|\nabla H(t, x)| \leq f(t) q(|x|)+g(t)
$$

for all $x \in R^{N}$ and a.e. $t \in[0, T]$;

(F2) there exists a positive function $q \in C\left(R^{+}, R^{+}\right)$which satisfies the conditions (q1)-(q4) and

$$
\liminf _{|x| \rightarrow \infty} \frac{1}{Q(|x|)} \int_{0}^{T} F(t, x) d t>0
$$

(F3) there exists $r<4 \pi^{2} / T^{2}$ such that

$$
(\nabla G(x)-\nabla G(y), x-y) \geq-r|x-y|^{2}
$$

for all $x, y \in R^{N}$.

Then problem (1) has at least one solution which minimizes the functional $\varphi$ on $H_{T}^{1}$ given by

$$
\varphi(u)=\frac{1}{2} \int_{0}^{T}|\dot{u}(t)|^{2} d t+\int_{0}^{T}[F(t, u(t))-F(t, 0)] d t
$$

for $u \in H_{T}^{1}$, where

$$
H_{T}^{1}=\left\{u:[0, T] \rightarrow R^{N} \mid u \text { is absolutely continuous, } u(0)=u(T) \text { and } \dot{u} \in L^{2}\left(0, T ; R^{N}\right)\right\}
$$

is a Hilbert space with the norm defined by

$$
\|u\|=\left(\int_{0}^{T}|u(t)|^{2} d t+\int_{0}^{T}|\dot{u}(t)|^{2} d t\right)^{1 / 2}
$$

for $u \in H_{T}^{1}$ 
Remark 1 Theorem 1.1 extends Theorem 1.1 in [1], in which it is a special case of our Theorem 1.1 corresponding to $G(x) \equiv 0$. There are functions $F(t, x)$ satisfying the assumptions of our Theorem 1.1 and not satisfying the corresponding assumptions in [1-20]. For example, let

$$
F(t, x)=G(x)+H(t, x)
$$

with $G(x)=C(x)-(r / 2)|x|^{2}$, which is bounded from below, and

$$
H(t, x)=f(t) \frac{|x|^{2}}{\ln \left(100+|x|^{2}\right)},
$$

where $C(x)$ is convex in $R^{N}\left(e . g\right.$., $\left.C(x)=(r / 2)\left(\left|x_{1}\right|^{4}+\left|x_{2}\right|^{2}+\cdots+\left|x_{N}\right|^{2}\right)\right), r<4 \pi^{2} / T^{2}, f(t) \in$ $L^{1}\left(0, T ; R^{N}\right)$ and $f(t)>0$ for a.e. $t \in[0, T]$. Let

$$
q(t)=\frac{t}{\ln \left(100+t^{2}\right)}, \quad C=0, \quad C^{*}=1 .
$$

Then $Q(t)=\int_{0}^{t} \frac{s}{\ln \left(100+s^{2}\right)} d s$, and it is easy to see the (F1), (F2), and (F3) conditions are satisfied. Then by Theorem 1.1, we conclude that problem (1) has at least one solution which minimizes the functional $\varphi$ in $H_{T}^{1}$. We note that $F$ does not satisfy those of the results given in [1-20] (e.g., $F$ does not satisfy (S1) of Theorem 1.1 in [1, 3], (ii) of Theorem 2 in [2], (8) of Theorem 2 in [5] and (4) of Theorem 1 in [9],..).

Replacing (3) with the following condition:

$$
\limsup _{|x| \rightarrow \infty} \frac{1}{Q(|x|)} \int_{0}^{T} F(t, x) d t<0,
$$

we then obtain Theorem 1.2 by the Saddle Point Theorem (see Theorem 4.6 in [13]).

Theorem 1.2 Suppose that $F(t, s)=G(x)+H(t, x)$ satisfies assumption (A), (F1), (F3), and (5). Assume that there exist $M \geq 0, N \geq 0$ such that

$$
|\nabla G(x)-\nabla G(y)| \leq M|x-y|+N
$$

for all $x, y \in R^{N}$. The problem (1) has at least one solution in $H_{T}^{1}$.

Remark 2 We note that Theorem 1.2 generalizes Theorem 1.2 in [1], which is the special case of our Theorem 1.2 corresponding to $G(x) \equiv 0$. There are functions $F(t, x)$ satisfying the assumptions of our Theorem 1.2, but not satisfying the corresponding assumptions in [1-20]. For example, let

$$
F(t, x)=G(x)+H(t, x)
$$

with $G(x)=C(x)-(r / 2)|x|^{2}$, which is bounded from above, and

$$
H(t, x)=\left(\frac{1}{3} T-t\right) \frac{|x|^{2}}{\ln \left(100+|x|^{2}\right)}+(d(t), x),
$$


where $C(x)$ satisfies the requirement that $\nabla C(x)$ is Lipschitz continuous and monotone in $R^{N}\left(e . g ., C(x)=(r / 2)\left(x_{1}+\left|x_{2}\right|^{2}+\cdots+\left|x_{N}\right|^{2}\right)\right), r<4 \pi^{2} / T^{2}$ and $d(t) \in L^{1}\left(0, T ; R^{N}\right)$. Take

$$
q(t)=\frac{t}{\ln \left(100+t^{2}\right)}, \quad Q(t)=\int_{0}^{t} \frac{s}{\ln \left(100+s^{2}\right)} d s,
$$

then it is easy to see that $F$ satisfies the assumptions of Theorem 1.2, we conclude that problem (1) has one solution in $H_{T}^{1}$. However, the results in [1-20] cannot be applied.

Theorem 1.3 Let the hypotheses of Theorem 1.2 be satisfied. Again, assume that there exist $\delta>0, \varepsilon>0$ and an integer $k>0$ such that

$$
-\frac{1}{2}(k+1)^{2} \omega^{2}|x|^{2} \leq F(t, x)-F(t, 0)
$$

for all $x \in R^{N}$ and a.e. $t \in[0, T]$, and

$$
F(t, x)-F(t, 0) \leq-\frac{1}{2} k^{2} \omega^{2}(1+\varepsilon)|x|^{2}
$$

for all $|x| \leq \delta$ and a.e. $t \in[0, T]$, where $\omega=\frac{2 \pi}{T}$. Then problem (1) has at least one non-trivial solution in $H_{T}^{1}$.

Theorem 1.4 Let the hypotheses of Theorem 1.1 be satisfied. Again, assume that there exist $\delta>0$ and an integer $k>0$ such that

$$
-\frac{1}{2}(k+1)^{2} \omega^{2}|x|^{2} \leq F(t, x)-F(t, 0) \leq-\frac{1}{2} k^{2} \omega^{2}|x|^{2}
$$

for all $|x| \leq \delta$ and a.e. $t \in[0, T]$. Then problem (1) has at least two non-trivial solutions in $H_{T}^{1}$.

\section{Preliminaries}

For $u \in H_{T}^{1}$, let

$$
\bar{u}=\frac{1}{T} \int_{0}^{T} u(t) d t, \quad \tilde{u}(t)=u(t)-\bar{u} .
$$

Then one has

$$
\|\tilde{u}\|_{\infty}^{2} \leq \frac{T}{12} \int_{0}^{T}|\dot{u}(t)|^{2} d t \quad \text { (Sobolev's inequality) }
$$

and

$$
\int_{0}^{T}|\tilde{u}(t)|^{2} d t \leq \frac{T^{2}}{4 \pi^{2}} \int_{0}^{T}|\dot{u}(t)|^{2} d t \quad \text { (Wirtinger's inequality), }
$$

where $\|\tilde{u}\|_{\infty}=\max _{t \in[0, T]}|u(t)|$.

It follows from assumption (A) that the corresponding function $\varphi$ on $H_{T}^{1}$ given by

$$
\varphi(u)=\frac{1}{2} \int_{0}^{T}|\dot{u}(t)|^{2} d t+\int_{0}^{T}[F(t, u(t))-F(t, 0)] d t
$$


is continuously differentiable and weakly lower semi-continuous on $H_{T}^{1}(c f$. [13], pp.12-13). Moreover, one has

$$
\left(\varphi^{\prime}(u), v\right)=\int_{0}^{T}(\dot{u}(t), \dot{v}(t)) d t+\int_{0}^{T}(\nabla F(t, u(t)), v(t)) d t
$$

for all $u, v \in H_{T}^{1}$. It is well known that the solutions to problem (1) correspond to the critical point of $\varphi$.

In order to prove our main theorems, we need the following lemmas.

Lemma 2.1 (Lemma 2.1 of [1]) Suppose that there exists a positive function $q$ which satisfies the conditions (q1), (q3), (q4) of (F1), then we have the following estimates:

(a) $0<q(t) \leq \varepsilon t+C_{0}, \forall \varepsilon>0, C_{0}>0, t \in R^{+}$,

(b) $\frac{q^{2}(t)}{Q(t)} \rightarrow 0$ as $t \rightarrow+\infty$,

(c) $Q(t) \rightarrow+\infty$ as $t \rightarrow+\infty$.

Lemma 2.2 (Theorem 4 of [11]) Let $X$ be a Banach space with a direct sum decomposition $X=X_{1} \oplus X_{2}$ with $\operatorname{dim} X_{2}<\infty$, and let $\varphi$ be a $C^{1}$ function on $X$ with $\varphi(0)=0$, satisfying (P.S.). Assume that for some $R>0$

$$
\varphi(u) \geq 0 \quad \text { for } u \in X_{1} \text { with }\|u\| \leq R
$$

and

$$
\varphi(u) \leq 0 \quad \text { for } u \in X_{2} \text { with }\|u\| \leq R .
$$

Again, assume that $\varphi$ is bounded from below and $\inf \varphi<0$. Then $\varphi$ has at least two non-zero critical points.

\section{The proof of main results}

For the sake of convenience, we will denote various positive constants as $C_{i}, i=1,2,3, \ldots$. Now, we are ready to prove our main result, Theorem 1.1.

Proof of Theorem 1.1 It follows from (F1), Lemma 2.1, and Sobolev's inequality that

$$
\begin{aligned}
& \left|\int_{0}^{T}[H(t, u(t))-H(t, \bar{u})] d t\right| \\
& \quad=\left|\int_{0}^{T} \int_{0}^{1}(\nabla H(t, \bar{u}+s \tilde{u}(t)), \tilde{u}(t)) d s d t\right| \\
& \quad \leq \int_{0}^{T} \int_{0}^{1} f(t) q(|\bar{u}+s \tilde{u}(t)|)|\tilde{u}(t)| d s d t+\int_{0}^{T} \int_{0}^{1} g(t)|\tilde{u}(t)| d s d t \\
& \quad \leq \int_{0}^{T} \int_{0}^{1} f(t)[q(|\bar{u}|+|\tilde{u}(t)|)+C]|\tilde{u}(t)| d s d t+\|\tilde{u}\|_{\infty} \int_{0}^{T} g(t) d t \\
& \quad \leq \int_{0}^{T} \int_{0}^{1} f(t)\left[C^{*}(q(|\bar{u}|)+q(|\tilde{u}(t)|))+C\right]|\tilde{u}(t)| d s d t+\|\tilde{u}\|_{\infty} \int_{0}^{T} g(t) d t \\
& \quad \leq C^{*}[q(|\bar{u}|)+q(|\tilde{u}(t)|)]\|\tilde{u}\|_{\infty} \int_{0}^{T} f(t) d t+C\|\tilde{u}\|_{\infty} \int_{0}^{T} f(t) d t+\|\tilde{u}\|_{\infty} \int_{0}^{T} g(t) d t
\end{aligned}
$$




$$
\begin{aligned}
\leq & C^{*}\left[\frac{3\left(4 \pi^{2}-r T^{2}\right)}{4 \pi^{2} C^{*} T}\|\tilde{u}\|_{\infty}^{2}+\frac{4 \pi^{2} C^{*} T}{3\left(4 \pi^{2}-r T^{2}\right)} q^{2}(|\bar{u}|)\left(\int_{0}^{T} f(t) d t\right)^{2}\right] \\
& +C^{*}\left[q\left(\|\tilde{u}\|_{\infty}\right)+C\right]\|\tilde{u}\|_{\infty} \int_{0}^{T} f(t) d t+C\|\tilde{u}\|_{\infty} \int_{0}^{T} f(t) d t+\|\tilde{u}\|_{\infty} \int_{0}^{T} g(t) d t \\
\leq & \frac{4 \pi^{2}-r T^{2}}{16 \pi^{2}} \int_{0}^{T}|\dot{u}(t)|^{2} d t+C_{1} q^{2}(|\bar{u}|)+C^{*}\left(\varepsilon\|\tilde{u}\|_{\infty}+C_{0}+C\right)\|\tilde{u}\|_{\infty} \int_{0}^{T} f(t) d t \\
& +C\|\tilde{u}\|_{\infty} \int_{0}^{T} f(t) d t+\|\tilde{u}\|_{\infty} \int_{0}^{T} g(t) d t \\
\leq & \left(\frac{4 \pi^{2}-r T^{2}}{16 \pi^{2}}+\varepsilon C_{2}\right) \int_{0}^{T}|\dot{u}(t)|^{2} d t+C_{1} q^{2}(|\bar{u}|)+C_{3}\left(\int_{0}^{T}|\dot{u}(t)|^{2} d t\right)^{1 / 2}
\end{aligned}
$$

for $u \in H_{T}^{1}$. From (F3) and Wirtinger's inequality we obtain

$$
\begin{aligned}
\int_{0}^{T} & {[G(u(t)-G(\bar{u})] d t} \\
& =\int_{0}^{T} \int_{0}^{1}(\nabla G(\bar{u}+s \tilde{u}(t)), \tilde{u}(t)) d s d t \\
& =\int_{0}^{T} \int_{0}^{1}(\nabla G(\bar{u}+s \tilde{u}(t))-\nabla G(\bar{u}), \tilde{u}(t)) d s d t \\
& =\int_{0}^{T} \int_{0}^{1} \frac{1}{s}(\nabla G(\bar{u}+s \tilde{u}(t))-\nabla G(\bar{u}), s \tilde{u}(t)) d s d t \\
& \geq \int_{0}^{T} \int_{0}^{1} \frac{1}{s}\left(-r s^{2}|\tilde{u}(t)|^{2}\right) d s d t \\
& \geq-\frac{r T^{2}}{8 \pi^{2}} \int_{0}^{T}|\dot{u}(t)|^{2} d t
\end{aligned}
$$

for $u \in H_{T}^{1}$. Hence we have

$$
\begin{aligned}
\varphi(u)= & \frac{1}{2} \int_{0}^{T}|\dot{u}(t)|^{2} d t+\int_{0}^{T}[H(t, u(t))-H(t, \bar{u})] d t \\
& +\int_{0}^{T}[G(u(t))-G(\bar{u})] d t+\int_{0}^{T} F(t, \bar{u}) d t-\int_{0}^{T} F(t, 0) d t \\
\geq & \left(\frac{4 \pi^{2}-r T^{2}}{16 \pi^{2}}-\varepsilon C_{2}\right) \int_{0}^{T}|\dot{u}(t)|^{2} d t-C_{3}\left(\int_{0}^{T}|\dot{u}(t)|^{2} d t\right)^{1 / 2} \\
& +Q(|\bar{u}|)\left[\frac{1}{Q(|\bar{u}|)} \int_{0}^{T} F(t, \bar{u}) d t-C_{1} \frac{q^{2}(|\bar{u}|)}{Q(|\bar{u}|)}\right]-\int_{0}^{T} F(t, 0) d t .
\end{aligned}
$$

Taking into account Lemma 2.1 and (F2), one has

$$
Q(|\bar{u}|)\left[\frac{1}{Q(|\bar{u}|)} \int_{0}^{T} F(t, \bar{u}) d t-C_{1} \frac{q^{2}(|\bar{u}|)}{Q(|\bar{u}|)}\right] \rightarrow+\infty
$$

as $|\bar{u}| \rightarrow+\infty$. 
As $\|u\| \rightarrow+\infty$ if and only if $\left(|\bar{u}|^{2}+\int_{0}^{T}|\dot{u}(t)|^{2} d t\right)^{1 / 2} \rightarrow+\infty$, for $\varepsilon$ small enough, by (12) and (13) one deduces that

$$
\varphi(u) \rightarrow+\infty \quad \text { as }\|u\| \rightarrow+\infty .
$$

Hence, by the least action principle, the problem (1) has at least one solution which minimizes the functional $\varphi$ in $H_{T}^{1}$.

Proof of Theorem 1.2 First we prove that $\varphi$ satisfies the (P.S.) condition. Suppose that $\left\{u_{n}\right\} \subset H_{T}^{1}$ is a (P.S.) sequence of $\varphi$, that is,

$$
\varphi^{\prime}\left(u_{n}\right) \rightarrow 0 \quad \text { as } n \rightarrow \infty
$$

and $\left\{\varphi\left(u_{n}\right)\right\}$ is bounded. In a way similar to that the proof of Theorem 1.1 above, we have

$$
\begin{aligned}
\left|\int_{0}^{T}\left(\nabla H\left(t, u_{n}(t)\right), \tilde{u}_{n}(t)\right) d t\right| \leq & \left(\frac{4 \pi^{2}-r T^{2}}{16 \pi^{2}}+\varepsilon C_{2}\right) \int_{0}^{T}\left|\dot{u}_{n}(t)\right|^{2} d t+C_{1} q^{2}\left(\left|\bar{u}_{n}\right|\right) \\
& +C_{3}\left(\int_{0}^{T}\left|\dot{u}_{n}(t)\right|^{2} d t\right)^{1 / 2}
\end{aligned}
$$

and

$$
\int_{0}^{T}\left(\nabla G\left(u_{n}(t)\right), \tilde{u}_{n}(t)\right) d t \geq-\frac{r T^{2}}{4 \pi^{2}} \int_{0}^{T}\left|\dot{u}_{n}(t)\right|^{2} d t
$$

for all $n$. Hence one has

$$
\begin{aligned}
\left\|\tilde{u}_{n}\right\| \geq & \left|\left\langle\varphi^{\prime}\left(u_{n}\right), \tilde{u}_{n}\right\rangle\right| \\
\geq & \int_{0}^{T}\left|\dot{u}_{n}(t)\right|^{2} d t+\int_{0}^{T}\left(\nabla F\left(t, u_{n}(t)\right), \tilde{u}_{n}(t)\right) d t \\
\geq & \int_{0}^{T}\left|\dot{u}_{n}(t)\right|^{2} d t+\int_{0}^{T}\left(\nabla G\left(u_{n}(t)\right), \tilde{u}_{n}(t)\right) d t \\
& \quad-\left|\int_{0}^{T}\left(\nabla H\left(t, u_{n}(t)\right), \tilde{u}_{n}(t)\right) d t\right| \\
\geq & \left(\frac{3\left(4 \pi^{2}-r T^{2}\right)}{16 \pi^{2}}-\varepsilon C_{2}\right) \int_{0}^{T}\left|\dot{u}_{n}(t)\right|^{2} d t-C_{1} q^{2}\left(\left|\bar{u}_{n}\right|\right) \\
& \quad-C_{3}\left(\int_{0}^{T}\left|\dot{u}_{n}(t)\right|^{2} d t\right)^{1 / 2}
\end{aligned}
$$

for large $n$. On the other hand, it follows from Wirtinger's inequality that

$$
\left(\int_{0}^{T}\left|\dot{u}_{n}(t)\right| d t\right)^{1 / 2} \leq\left\|\tilde{u}_{n}\right\| \leq\left(\frac{T^{2}}{4 \pi^{2}}+1\right)^{1 / 2}\left(\int_{0}^{T}\left|\dot{u}_{n}(t)\right| d t\right)^{1 / 2}
$$

for large $n$. Combining (14) with (15), we obtain

$$
C_{4} q\left(\left|\bar{u}_{n}\right|\right) \geq\left(\int_{0}^{T}\left|\dot{u}_{n}(t)\right|^{2} d t\right)^{1 / 2}-C_{5}
$$


for all large $n$ and $\varepsilon$ small enough. It follows from (6), Cauchy-Schwarz's inequality, and Wirtinger's inequality that

$$
\begin{aligned}
\int_{0}^{T} & {\left[G\left(u_{n}(t)\right)-G\left(\bar{u}_{n}\right)\right] d t } \\
& =\int_{0}^{T} \int_{0}^{1}\left(\nabla G\left(\bar{u}_{n}+\tilde{s} u_{n}(t)\right), \tilde{u}_{n}(t)\right) d s d t \\
& =\int_{0}^{T} \int_{0}^{1} \frac{1}{s}\left(\nabla G\left(\bar{u}_{n}+\tilde{s} u_{n}(t)\right)-\nabla G\left(\bar{u}_{n}\right), s \tilde{u}_{n}(t)\right) d s d t \\
& \leq \int_{0}^{T} \int_{0}^{1}\left(s M\left|\tilde{u}_{n}(t)\right|^{2}+N\left|\tilde{u}_{n}(t)\right|\right) d s d t \\
& \leq \frac{M}{2} \int_{0}^{T}\left|\tilde{u}_{n}(t)\right|^{2} d t+N \sqrt{T}\left(\int_{0}^{T}\left|\tilde{u}_{n}(t)\right|^{2} d t\right)^{1 / 2} \\
& \leq \frac{M T^{2}}{8 \pi^{2}} \int_{0}^{T}\left|\dot{u}_{n}(t)\right|^{2} d t+\frac{N T \sqrt{T}}{2 \pi}\left(\int_{0}^{T}\left|\dot{u}_{n}(t)\right|^{2} d t\right)^{1 / 2}
\end{aligned}
$$

for all $n$. By the proof Theorem 1.1 we have

$$
\begin{aligned}
\left|\int_{0}^{T}\left[H\left(t, u_{n}(t)\right)-H\left(t, \bar{u}_{n}\right)\right] d t\right| \leq & \left(\frac{4 \pi^{2}-r T^{2}}{16 \pi^{2}}+\varepsilon C_{2}\right) \int_{0}^{T}\left|\dot{u}_{n}(t)\right|^{2} d t \\
& +C_{1} q^{2}\left(\left|\bar{u}_{n}\right|\right)+C_{3}\left(\int_{0}^{T}\left|\dot{u}_{n}(t)\right|^{2} d t\right)^{1 / 2}
\end{aligned}
$$

for all $n$. By (16), (17), (18), Lemma 2.1, and (5), one has

$$
\begin{aligned}
\varphi\left(u_{n}\right)= & \frac{1}{2} \int_{0}^{T}\left|\dot{u}_{n}(t)\right|^{2} d t+\int_{0}^{T}\left[G\left(u_{n}(t)\right)-G\left(\bar{u}_{n}\right)\right] d t \\
& +\int_{0}^{T}\left[H\left(t, u_{n}(t)\right)-H\left(t, \bar{u}_{n}\right)\right] d t+\int_{0}^{T} F\left(t, \bar{u}_{n}\right) d t-\int_{0}^{T} F(t, 0) d t \\
\leq & \left(\frac{12 \pi^{2}-r T^{2}+2 M T^{2}}{16 \pi^{2}}+\varepsilon C_{2}\right) \int_{0}^{T}\left|\dot{u}_{n}(t)\right|^{2} d t+C_{1} q^{2}\left(\left|\bar{u}_{n}\right|\right) \\
& +\left(C_{3}+\frac{N T \sqrt{T}}{2 \pi}\right)\left(\int_{0}^{T}\left|\dot{u}_{n}(t)\right|^{2} d t\right)^{1 / 2}+\int_{0}^{T} F\left(t, \bar{u}_{n}\right) d t-\int_{0}^{T} F(t, 0) d t \\
\leq & C_{6}\left[C_{4} q\left(\left|\bar{u}_{n}\right|\right)+C_{5}\right]^{2}+C_{1} q^{2}\left(\left|\bar{u}_{n}\right|\right)+\left(C_{3}+\frac{N T \sqrt{T}}{2 \pi}\right)\left[C_{4} q\left(\left|\bar{u}_{n}\right|\right)+C_{5}\right] \\
& +\int_{0}^{T} F\left(t, \bar{u}_{n}\right) d t-\int_{0}^{T} F(t, 0) d t \\
\leq & C_{7} q^{2}\left(\left|\bar{u}_{n}\right|\right)+C_{8} q\left(\left|\bar{u}_{n}\right|\right)+C_{9}+\int_{0}^{T} F\left(t, \bar{u}_{n}\right) d t-\int_{0}^{T} F(t, 0) d t \\
\leq & Q\left(\left|\bar{u}_{n}\right|\right)\left[C_{7} \frac{q^{2}\left(\left|\bar{u}_{n}\right|\right)}{Q\left(\left|\bar{u}_{n}\right|\right)}+C_{8} \frac{q\left(\left|\bar{u}_{n}\right|\right)}{Q\left(\left|\bar{u}_{n}\right|\right)}+\frac{1}{Q\left(\left|\bar{u}_{n}\right|\right)} \int_{0}^{T} F\left(t, \bar{u}_{n}\right) d t\right] \\
& +C_{9}-\int_{0}^{T} F(t, 0) d t \\
\rightarrow & -\infty
\end{aligned}
$$

as $\left|\bar{u}_{n}\right| \rightarrow \infty$. This contradicts the boundedness of $\left\{\varphi\left(u_{n}\right)\right\}$. 
Thus $\left\{\bar{u}_{n}\right\}$ is bounded. Notice (16) and (a) of Lemma 2.1, $\left\{u_{n}\right\}$ is bounded, and by following the same arguments used as Proposition 4.1 in [13] we conclude that the (P.S.) condition is satisfied.

We now prove that $\varphi$ satisfies the other conditions of the Saddle Point Theorem. Let $\widetilde{H}_{T}^{1}$ be the subspace of $H_{T}^{1}$ given by

$$
\widetilde{H}_{T}^{1}=\left\{u \in H_{T}^{1} \mid \bar{u}=0\right\} .
$$

Then one has

$$
\varphi(u) \rightarrow+\infty
$$

as $|u| \rightarrow \infty$ in $\widetilde{H}_{T}^{1}$. In fact, by the proof of Theorem 1.1 we have

$$
\int_{0}^{T}[G(u(t))-G(0)] d t \geq-\frac{r T^{2}}{8 \pi^{2}} \int_{0}^{T}|\dot{u}(t)|^{2} d t
$$

for all $u \in \widetilde{H}_{T}^{1}$. In addition, by (F1), Sobolev's inequality and Lemma 2.1, we have

$$
\begin{aligned}
& \left|\int_{0}^{T}[H(t, u(t))-H(t, 0)] d t\right| \\
& \quad=\left|\int_{0}^{T} \int_{0}^{1}(\nabla H(t, s u(t)), u(t)) d s d t\right| \\
& \quad \leq \int_{0}^{T} f(t) q(|s u(t)|)|u(t)| d t+\int_{0}^{T} g(t)|u(t)| d t \\
& \quad \leq \int_{0}^{T} f(t)[q(|u(t)|)+C]|u(t)| d t+\|u\|_{\infty} \int_{0}^{T} g(t) d t \\
& \quad \leq \varepsilon\|u\|_{\infty}^{2} \int_{0}^{T} f(t) d t+\left(C_{0}+C\right)\|u\|_{\infty} \int_{0}^{T} f(t) d t+\|u\|_{\infty} \int_{0}^{T} g(t) d t \\
& \quad \leq \varepsilon C_{10} \int_{0}^{T}|\dot{u}(t)|^{2} d t+C_{11}\left(\int_{0}^{T}|\dot{u}(t)|^{2} d t\right)^{1 / 2} \cdot
\end{aligned}
$$

Hence one has

$$
\begin{aligned}
\varphi(u)= & \frac{1}{2} \int_{0}^{T}|\dot{u}(t)|^{2} d t+\int_{0}^{T}[G(u(t))-G(0)] d t \\
& +\int_{0}^{T}[H(t, u(t))-H(t, 0)] d t \\
& \geq\left(\frac{4 \pi^{2}-r T^{2}}{8 \pi^{2}}-\varepsilon C_{10}\right) \int_{0}^{T}|\dot{u}(t)|^{2} d t \\
& -C_{11}\left(\int_{0}^{T}|\dot{u}(t)|^{2} d t\right)^{1 / 2} .
\end{aligned}
$$

By Wirtinger's inequality, one has $\|u\| \rightarrow+\infty$ if and only if $\left(\int_{0}^{T}|\dot{u}(t)|^{2} d t\right)^{1 / 2} \rightarrow+\infty$ on $\tilde{H}_{T}^{1}$. Hence, for $\varepsilon$ small enough, this implies (19) by (15) and (20). 
On the other hand, by (5) and Lemma 2.1, we get

$$
\varphi(x)=\int_{0}^{T} F(t, x) d t-\int_{0}^{T} F(t, 0) d t \rightarrow-\infty
$$

as $|x| \rightarrow \infty$ in $R^{N}$.

Now, Theorem 1.2 is proved by (19), (21), and the application of the Saddle Point Theorem.

Proof of Theorem 1.3 Let $E=H_{T}^{1}$,

$$
H_{k}=\left\{\sum_{j=0}^{k}\left(a_{j} \cos j \omega t+b_{j} \sin j \omega t\right) \mid a_{j}, b_{j} \in R^{N}, j=0,1, \ldots, k\right\}
$$

and

$$
\psi(u)=-\varphi(u)=-\frac{1}{2} \int_{0}^{T}|\dot{u}(t)|^{2} d t-\int_{0}^{T}[F(t, x)-F(t, 0)] d t .
$$

Then $\psi \in C^{1}(E, R)$ satisfies the (P.S.) condition by the proof of Theorem 1.2. In view of Theorem 5.29 and Example 5.26 in [21] (e.g., the Generalized Mountain Pass Theorem), we only need to prove that

$\left(\psi_{1}\right) \liminf \|u\|^{-2} \psi(u)>0$ as $u \rightarrow 0$ in $H_{k}$,

$\left(\psi_{2}\right) \psi(u) \leq 0$ for all $u \in H_{k}^{\perp}$, and

$\left(\psi_{3}\right) \psi(u) \rightarrow-\infty$ as $\|u\| \rightarrow \infty$ in $H_{k-1}^{\perp}$.

By (F1) and Lemma 2.1, one has

$$
\begin{aligned}
H(t, x)-H(t, 0) & =\int_{0}^{1}(\nabla H(t, s x), x) d s \\
& \leq \int_{0}^{1}(f(t) q(|s x|)+g(t), x) d s \\
& \leq f(t)[q(|x|)+C]|x|+g(t)|x| \\
& \leq f(t)\left(\varepsilon|x|+C_{0}+C\right)|x|+g(t)|x| \\
& =\varepsilon f(t)|x|^{2}+\left[\left(C_{0}+C\right) f(t)+g(t)\right]|x|
\end{aligned}
$$

for all $|x| \geq \delta$ and a.e. $t \in[0, T]$. From (6), it follows that

$$
\begin{aligned}
G(x)-G(0) & =\int_{0}^{1}(\nabla G(s x), x) d s \\
& =\int_{0}^{1}(\nabla G(s x)-\nabla G(0), x) d s \\
& \leq \int_{0}^{1}(s M|x|+N)|x| d s \\
& \leq M|x|^{2}+N|x|
\end{aligned}
$$


for all $|x| \geq \delta$ and a.e. $t \in[0, T]$. From (23) and (24), we obtain

$$
\begin{aligned}
F(t, x)-F(t, 0) & \leq[\varepsilon f(t)+M]|x|^{2}+\left[\left(C_{0}+C\right) f(t)+g(t)+N\right]|x| \\
& \leq \gamma(t)|x|^{3}
\end{aligned}
$$

for all $|x| \geq \delta$, a.e. $t \in[0, T]$ and some $\gamma(t) \in L^{1}\left(0, T ; R^{+}\right)$given by

$$
\gamma(t)=[\varepsilon f(t)+M] \delta^{-1}+\left[\left(C_{0}+C\right) f(t)+g(t)+N\right] \delta^{-2} .
$$

Now, it follows from (8) that

$$
F(t, x)-F(t, 0) \leq-\frac{1}{2} k^{2} \omega^{2}(1+\varepsilon)|x|^{2}+\gamma(t)|x|^{3}
$$

for all $x \in R^{N}$ and a.e. $t \in[0, T]$. Hence, we obtain

$$
\begin{aligned}
\psi(u) & \geq-\frac{1}{2} \int_{0}^{T}|\dot{u}(t)|^{2} d t+\frac{1}{2} k^{2} \omega^{2}(1+\varepsilon) \int_{0}^{T}|u(t)|^{2} d t-\int_{0}^{T} \gamma(t)|u(t)|^{3} \\
& \geq \frac{1}{2} \varepsilon \int_{0}^{T}|\dot{u}(t)|^{2} d t+\frac{1}{2} k^{2} \omega^{2}(1+\varepsilon)|\bar{u}|^{2} T-\|u\|_{\infty}^{3} \int_{0}^{T} \gamma(t) d t \\
& \geq C_{12}\|u\|^{2}-C_{13}\|u\|^{3}
\end{aligned}
$$

for all $u \in H_{k}$. Then $\left(\psi_{1}\right)$ follows from the above inequality. For $u \in H_{k}^{\perp}$, by (7), one has

$$
\psi(u) \leq-\frac{1}{2} \int_{0}^{T}|\dot{u}(t)|^{2} d t+\frac{1}{2} k^{2} \omega^{2} \int_{0}^{T}|u(t)|^{2} d t \leq 0,
$$

which is $\left(\psi_{2}\right)$. Finally, $\left(\psi_{3}\right)$ follows from (21). Hence the proof of Theorem 1.3 is completed.

Proof of Theorem 1.4 From the proof of Theorem 1.1 we know that $\varphi$ is coercive, which implies that $\varphi$ satisfies the (P.S.) condition. In a manner similar to ones used by the literature of $[10,11]$, we can get the multiplicity results. For convenience of the readers, we give details.

Let $X_{2}$ be the finite-dimensional subspace $H_{k}$ given by (22) and let $X_{1}=X_{2}^{\perp}$. Then by (9) we have

$$
\varphi(u) \leq \frac{1}{2} \int_{0}^{T}|\dot{u}(t)|^{2} d t-\frac{1}{2} k^{2} \omega^{2} \int_{0}^{T}|u(t)|^{2} d t \leq 0
$$

for all $u \in X_{2}$ with $\|u\| \leq C_{14}^{-1} \delta$ and

$$
\varphi(u) \geq \frac{1}{2} \int_{0}^{T}|\dot{u}(t)|^{2} d t-\frac{1}{2}(k+1)^{2} \omega^{2} \int_{0}^{T}|u(t)|^{2} d t \geq 0
$$

for all $u \in X_{1}$ with $\|u\| \leq C_{14}^{-1} \delta$, where $C_{14}$ is the positive constant such that $\|u\|_{\infty} \leq C_{14}\|u\|$ for all $u \in H_{T}^{1}$. 
The case that

$$
\int_{0}^{T}[F(t, x)-F(t, 0)] d t<0
$$

for some $|x|<\delta$, implies inf $\varphi<0$. Now our Theorem 1.4 follows from Lemma 2.2.

On the contrary, we have

$$
\int_{0}^{T}[F(t, x)-F(t, 0)] d t \geq 0
$$

for all $|x|<\delta$. Then it follows from (9) that for every given $|x|<\delta$ one has

$$
F(t, x)-F(t, 0)=0
$$

for a.e. $t \in[0, T]$. Let

$$
E(x)=\{t \in[0, T] \mid F(t, x)-F(t, 0) \neq 0\} .
$$

Then meas $E(x)=0$ for all $|x|<\delta$. Given $\left|x_{0}\right|<\delta$ we have

$$
\left|x_{0}+\frac{1}{n} e_{m}\right|<\delta
$$

for $n>\frac{1}{\delta-\left|x_{0}\right|}$, where $\left\{e_{m} \mid 1<m<N\right\}$ is the canonical basis of $R^{N}$. Thus we obtain

$$
\left(\nabla F\left(t, x_{0}\right), e_{m}\right)=\lim _{n \rightarrow \infty} \frac{F\left(t, x_{0}+\frac{1}{n} e_{m}\right)-F\left(t, x_{0}\right)}{\frac{1}{n}}=0
$$

for all $t \notin\left(\bigcup\left\{E\left(x_{0}+\frac{1}{n} e_{m}\right) \mid n>\frac{1}{\delta-\left|x_{0}\right|}, 1 \leq m \leq N\right\} \cup E\left(x_{0}\right)\right)$, which implies that $\nabla F\left(t, x_{0}\right)=0$ for a.e. $t \in[0, T]$, that is, $x_{0}$ is a solution to problem (1). Hence all $|x|<\delta$ are the solutions to problem (1). Therefore, Theorem 1.4 is proved.

\section{Competing interests}

The authors declare that they have no competing interests.

\section{Authors' contributions}

All authors contributed equally to the writing of this paper. All authors read and approved the final manuscript.

\section{Author details}

'School of Sciences, Guizhou Minzu University, Guiyang, 550025, China. ${ }^{2}$ School of Digital Media, Jiangnan University, Wuxi, Jiangsu 214122, China. ${ }^{3}$ School of Mathematics and Computer Science, Bijie University, Bijie, 551700, China.

\section{Acknowledgements}

All authors were supported by Science and Technology Foundation of the Guizhou Province (no. LKM[2011]31; no. LKB[2012]19; no. [2013]2141). The second author was also supported by the Six Talent Peaks Project of Jiangsu Province (No. DZXX-028). The authors would like also to thank the referees for their valuable suggestions and comments which improve the exposition of this paper. 


\section{References}

1. Wang, ZY, Zhang, JH: Periodic solutions for nonautonomous second order Hamiltonian systems with sublinear nonlinearity. Bound. Value Probl. 2011, 23 (2011). doi:10.1186/1687-2770-2011-23

2. Aizmahin, N, An, TQ: The existence of periodic solutions of non-autonomous second-order Hamiltonian systems Nonlinear Anal. 74, 4862-4867 (2011)

3. Wang, ZY, Zhang, JH: Periodic solutions of a class of second order non-autonomous Hamiltonian systems. Nonlinear Anal. 72, 4480-4487 (2010)

4. Wang, Z, Zhang, J: Periodic solutions of non-autonomous second order Hamiltonian systems with $p$-Laplacian. Electron. J. Differ. Equ. 2009, 17 (2009)

5. Ye, YW, Tang, CL: Periodic solutions for some non-autonomous second order Hamiltonian systems. J. Math. Anal. Appl. 344, 462-471 (2008)

6. Schechter, M: Periodic non-autonomous second-order dynamical systems. J. Differ. Equ. 223(2), 290-302 (2006)

7. Luan, SX, Mao, AM: Periodic solutions of nonautonomous second order Hamiltonian systems. Acta Math. Appl. Sinica (Engl. Ser.) 21(4), 685-690 (2005)

8. $\mathrm{Wu}, \mathrm{X}$ : Saddle point characterization and multiplicity of periodic solutions of non-autonomous second order systems. Nonlinear Anal. TMA 58, 899-907 (2004)

9. Ma, J, Tang, CL: Periodic solutions for some nonautonomous second-order systems. J. Math. Anal. Appl. 275(2), 482-494 (2002)

10. Tang, CL: Periodic solutions for nonautonomous second systems with sublinear nonlinearity. Proc. Am. Math. Soc 126(11), 3263-3270 (1998)

11. Brezis, H, Nirenberg, L: Remarks on finding critical points. Commun. Pure Appl. Math. 44, 939-963 (1991)

12. Ahmad, S, Lazer, AC: Critical point theory and a theorem of Amral and Pea. Boll. Unione Mat. Ital., B 3, 583-598 (1984)

13. Mawhin, J, Willem, M: Critical Point Theory and Hamiltonian Systems. Springer, New York (1989)

14. Tang, CL: Periodic solutions of nonautonomous second order systems with $\gamma$-quasisubadditive potential. J. Math. Anal. Appl. 189, 671-675 (1995)

15. Tang, CL: Existence and multiplicity of periodic solutions for nonautonomous second order systems. Nonlinear Anal. 32, 299-304 (1998)

16. Long, YM: Nonlinear oscillations for classical Hamiltonian systems with bi-even subquadratic potentials. Nonlinear Anal. 24, 1665-1671 (1995)

17. Mawhin, J: Semi-coercive monotone variational problems. Bull. Cl. Sci., Acad. R. Belg. 73, 118-130 (1987)

18. Berger, MS, Schechter, M: On the solvability of semilinear gradient operator equations. Adv. Math. 25, 97-132 (1977)

19. Tang, CL: Periodic solutions of nonautonomous second order systems. J. Math. Anal. Appl. 202, $465-469$ (1996)

20. Willem, M: Oscillations forcées de systèmes hamiltoniens. In: Public Sémin. Analyse Non Linéaire. Univ. Besancon (1981)

21. Rabinowitz, PH: Minimax Methods in Critical Point Theory with Applications to Differential Equations. CBMS Reg. Conf. Ser. Math., vol. 65. Am. Math. Soc., Providence (1986)

10.1186/1029-242X-2014-411

Cite this article as: Suo et al.: Existence and multiplicity of periodic solutions for some second-order Hamiltonian systems. Journal of Inequalities and Applications 2014, 2014:411

\section{Submit your manuscript to a SpringerOpen ${ }^{\circ}$ journal and benefit from:}

- Convenient online submission

- Rigorous peer review

- Immediate publication on acceptance

- Open access: articles freely available online

- High visibility within the field

- Retaining the copyright to your article 